\title{
Contribución al conocimiento de la biota liquénica de la Reserva Nacional Katalalixar, Patagonia, Chile
}

\section{Contribution to the knowledge of the lichen biota of Katalalixar National Reserve, Patagonia, Chile}

\section{Johana Villagra ${ }^{1, *}$, David Alors ${ }^{2}$ \& Angélica Casanova-Katny ${ }^{1,3}$}

\author{
${ }^{1}$ Laboratorio de Ecofisiología Vegetal, Facultad de Recursos Naturales, Universidad Católica de Temuco, Campus Luis Rivas del Canto, Rudecindo Ortega 03694, Temuco, Chile. \\ ${ }^{2}$ Microalgal Biotechnology Laboratory, Blaustein Institute for Desert Research, Ben-Gurion University of the Negev, Sede-Boker Campus 84990, Israel. \\ ${ }^{3}$ Núcleo de Estudios Ambientales, Universidad Católica de Temuco, Rudecindo Ortega 02950, Temuco, Chile. \\ *E-mail: jovyvillagra@gmail.com
}

\section{RESUMEN}

Los líquenes son reconocidos como biondicadores de procesos ecosistémicos asociados a disturbios ambientales. Sin embargo, su evaluación en el tiempo requiere de registros sistemáticos de biodiversidad los cuales son inexistentes en la Reserva Nacional Katalalixar. El objetivo de este estudio ha sido catalogar la riqueza y distribución de la biota liquénica de la Reserva Nacional Katalalixar, no solo identificando las especies presentes, sino interpretándolas en su contexto ecológico y biogeográfico. El muestreo fue realizado al azar, en bosques de Nothofagus nitida, Pilgerodendron uviferum y en turberas. Se identificaron 70 especies, pertenecientes a 32 familias y 65 géneros. Se encontró un elevado número de endemismo, el 34\% de las especies son endémicas del sur de Sudamérica, y además se suma el endemismo chileno Pseudocyphellaria guzmanii constituyendo un nuevo registro para la región de Aysén.

Palabras clave: endemismo, Katalalixar, líquenes.

\section{ABSTRACT}

Lichens are recognized as bioindicators of ecosystem processes associated with environmental disturbances. However, its evaluation in the time requires systematic records of biodiversity which are non-existent in the Katalalixar National Reserve. The aim of this study was to catalog the richness and distribution of lichen biota of Katalalixar National Reserve, not only by identifying the species but interpreting them in their ecological and biogeographic context. By random sampling in Nothofagus nitida, Pilgerodendron uviferum forests and peat bogs 70 species were identified, belonging to 32 families and 65 genera. A high number of endemism was found, with $34 \%$ of the species endemic to Southern South American and the Chilean endemic Pseudocyphellaria guzmanii constitutes a new record for the Aisen Region.

Keywords: endemism, Katalalixar, lichens.

\section{INTRODUCCIÓN}

Los líquenes son un grupo biológico importante de los ecosistemas ya que contribuyen a la diversidad biológica y a la conservación de ésta, por ejemplo, albergan diversas y únicas comunidades microbianas e invertebrados, proporcionan alimentos y material de anidación para invertebrados y vertebrados y aportan carbono y nitrógeno a los ciclos de nutrientes (Kuusinen \& Siitonen 1998; Pharo et al. 1999; Arnold et al. 2009; Hodkinson \& Lutzoni 2009; Rubio et 
al. 2013; Asplund \& Wardle 2016). Las singularidades del aislamiento geográfico del territorio chileno han generado una alta proporción de endemismos en todos los grupos biológicos incluyendo los líquenes (Galloway 1991, 1996; Villagrán \& Hinojosa 1997). Los líquenes endémicos contribuyen especialmente a incrementar la biodiversidad y debido a su distribución más restringida son más vulnerables frente a la pérdida de área climáticamente adecuada (Allen \& Lendemer 2016). El uso de rasgos funcionales y atributos ecológicos pueden mejorar la interpretación de cómo las especies responden al cambio, por ejemplo, un estudio reciente sugirió que los macrolíquenes con cianobacterias son el grupo de líquenes más amenazados por el impacto futuro del clima (Sillett 1994; Richardson \& Cameron 2004; Rubio-Salcedo et al. 2017). Entre las principales amenazas que afectan a los líquenes se encuentra la contaminación atmosférica, explotación forestal, pérdida y fragmentación del hábitat (especialmente en líquenes epífitos), incendios (principalmente los que involucren bosques nativos maduros) y el cambio climático (Martínez et al. 2003; Ellis 2013; Lendemer \& Allen 2014; Casanova-Katny et al. 2019).

Los líquenes como organismos poiquilohídricos han sido utilizados como bioindicadores ya que resultan especialmente sensibles a una amplia gama de factores ambientales (McCune 2000; Hedenäs \& Ericson 2000; Goirdani \& Brunialti 2015) que pueden dar lugar incluso a la desaparición de especies sensibles (Humphrey et al. 2002; Paillet et al. 2010). Sin embargo, para evaluar el estado de conservación de un área, utilizando líquenes como bioindicadores, se requieren antecedentes previos de la diversidad, el comportamiento de estos organismos y la historia de las zonas muestreadas frente a la perturbación (Jentsch et al. 2002; Rivas-Plata et al. 2008; Gatica et al. 2011). En este sentido, la Reserva Nacional Katalalixar (RN Katalalixar) se caracteriza por varios biomas en buen estado de conservación (Seremi MMAAysén 2018) y con un número considerable de macro y microhábitats disponibles para la exitosa colonización de líquenes. Sin embargo, existe escasa información respecto a estos organismos en esta reserva (Quilhot et al. 2012). Considerando los datos previos, y dado que la conservación de los bosques maduros es una de las líneas de gestión dentro de este espacio protegido (Seremi MMA-Aysén 2018) se ha propuesto un estudio preliminar de la biota liquénica, centrada principalmente en los bosques de Nothofagus nitida (Phil.) Krasser, Pilgerodendron uviferum (D. Don) Florin y en turberas. El objetivo de este estudio es contribuir al conocimiento de la biota liquénica y su distribución en la RN Katalalixar. Para ello, se propuso; a) identificar las especies de líquenes en el área estudiada y b) proporcionar información sobre la distribución y datos ecológicos de las especies en el área estudiada.

\section{MATERIALES Y MÉTODOS}

\section{Área de estudio}

La Reserva Nacional Katalalixar (Fig. 1) está ubicada en la comuna de Tortel en la Región de Aysén (47046,45' S / $73^{\circ} 59,24^{\prime}$ O). El clima es oceánico de tipo templado húmedo según Köppen (Scherhag 1969). Amigo \& Ramírez (1998) lo definen como bioclima supratemperado de ombro tipo ultraperhúmedo. La media anual de precipitaciones fluctúa entre los 3.500 a $4.000 \mathrm{~mm}$, con todos los meses del año húmedos y su temperatura media anual es de $7{ }^{\circ} \mathrm{C}$ (Hajek Di Castri 1975).

\section{Metodología}

La topografía de la reserva y la ausencia de comunicaciones terrestres provocan el difícil acceso y sesgó los puntos de muestreo a zonas muy próximas al borde costero con alturas sobre el nivel del mar que no superaron los 5 metros. Se seleccionaron 9 puntos de muestreo de forma aleatoria a fin de encontrar la máxima diversidad de líquenes durante los días 24 y 27 de abril de 2019 (Tabla 1). Se exploraron 6 puntos correspondientes a bosque de Nothofagus nitida (puntos

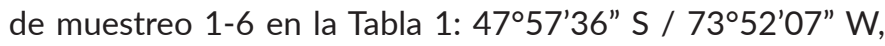
$47^{\circ} 56^{\prime} 25^{\prime \prime} \mathrm{S} / 73^{\circ} 57^{\prime} 46^{\prime \prime} \mathrm{W}, 47^{\circ} 56^{\prime} 25^{\prime \prime} \mathrm{S} / 74^{\circ} 11^{\prime} 47^{\prime \prime} \mathrm{W}$, $47^{\circ} 57^{\prime} 15^{\prime \prime} \mathrm{S} / 7^{\circ} 14^{\prime} 57^{\prime \prime} \mathrm{W}, 47^{\circ} 54^{\prime} 33^{\prime \prime} \mathrm{S} / 7^{\circ} 18^{\prime} 53^{\prime \prime} \mathrm{W}$, $47^{\circ} 51^{\prime} 16^{\prime \prime} \mathrm{S} / 7^{\circ} 25^{\prime} 02^{\prime \prime}$ W) con un sotobosque denso y sombrío. Corresponde a un bosque higromórfico de lugares fríos y húmedos con influencia euhiperoceánico (Luebert \& Pliscoff 2006). Un bosque de Pilgerodendron uviferum asociado a una turbera de Sphagnum magellanicum Brid. (Pon Pon) y expuesto a mayor exposición a la luz (Punto de muestreo 7, 47 $\left.46^{\prime} 45^{\prime \prime} \mathrm{S} / 7^{\circ} 59^{\prime} 23^{\prime \prime} \mathrm{W}\right)$. Finalmente, 2 ambientes de turberas mixtas (Puntos de muestreo 8-9, 47 $57^{\prime} 57^{\prime \prime} \mathrm{S} /$ $73^{\circ} 50^{\prime} 19^{\prime \prime} \mathrm{W}, 47^{\circ} 57^{\prime} 36^{\prime \prime} \mathrm{S} / 7^{\circ} 52^{\prime} 07^{\prime \prime} \mathrm{W}$ ) asociadas al musgo S. magellanicum dominadas por Desfontainia fulgens D. Don, Gaultheria mucronata (L. f.) Hook. et Arn. (chaura, murta), Embothrium coccineum J.R. Forst. et G. Forst.) (Notro), entre otras especies vasculares.

\section{DETERMINACIÓN TAXONÓMICA}

La identificación de especies de líquenes se hizo basada en la literatura específica de Galloway (1985, 1992a, 1994), Stenroos (1995), Wedin (1995), Bjerke et al. (2003), Bjerke (2005) y otros (Nash et al. 1995; Nash et al. 2002; Nash \& Elix 2002a; Nash \& Elix 2002b; Nash \& Elix 2002c). La nomenclatura sigue el Index Fungorum. Se siguió el sistema adoptado por Brodo (2001), que incluye la utilización de lupa estereoscópica para la medición de caracteres morfológicos externos y microscopio óptico para la observación de cortes al apotecio y secciones de talo. La identificación de los líquenes 
también incluyó test químicos (spot test) que determinan la presencia o ausencia de determinadas substancias en distintas estructuras del talo liquénico (Orange et al. 2001). Los reactivos utilizados para el test químico fueron $\mathrm{K}$ (solución saturada de $\mathrm{KOH}$ al 10\%), C (solución acuosa de hipoclorito de sodio al $5 \%$ ) y KC (aplicación de $\mathrm{K}$ seguida de $\mathrm{C}$ ). Parte del material recolectado ha sido depositado en el Herbario de la Universidad Católica de Temuco. La distribución biogeográfica se indica utilizando las siguientes cinco categorías: endémico, cosmopolita, austral, tropical y bipolar. En este trabajo se considerará como endemismo aquellas especies presentes únicamente en el sur de Sudamérica, la categoría austral incluye tanto neoaustral como paleoaustral y la categoría de tropical incluye tanto especies neotropicales como aquellas especies tropicales de distribución más amplia.
Las especies cuya distribución no se ajusta a ninguna de estas categorías se catalogan como "otros". Estas categorías son una simplificación de las utilizadas por Galloway (2008).

\section{RESULTADOS Y DISCUSIÓN}

La riqueza específica alcanzó un total de 68 especies de líquenes distribuidas en 32 familias y 65 géneros (Tabla 1, Fig. 2). Nuestros resultados complementan las 26 especies identificadas previamente por Quilhot et al. (2012) en la reserva, aumentando en un $180 \%$ la especies citadas y alcanzando el $21 \%$ de las especies y $39 \%$ de los géneros de líquenes descritos para la región, respectivamente (Fig. 2).
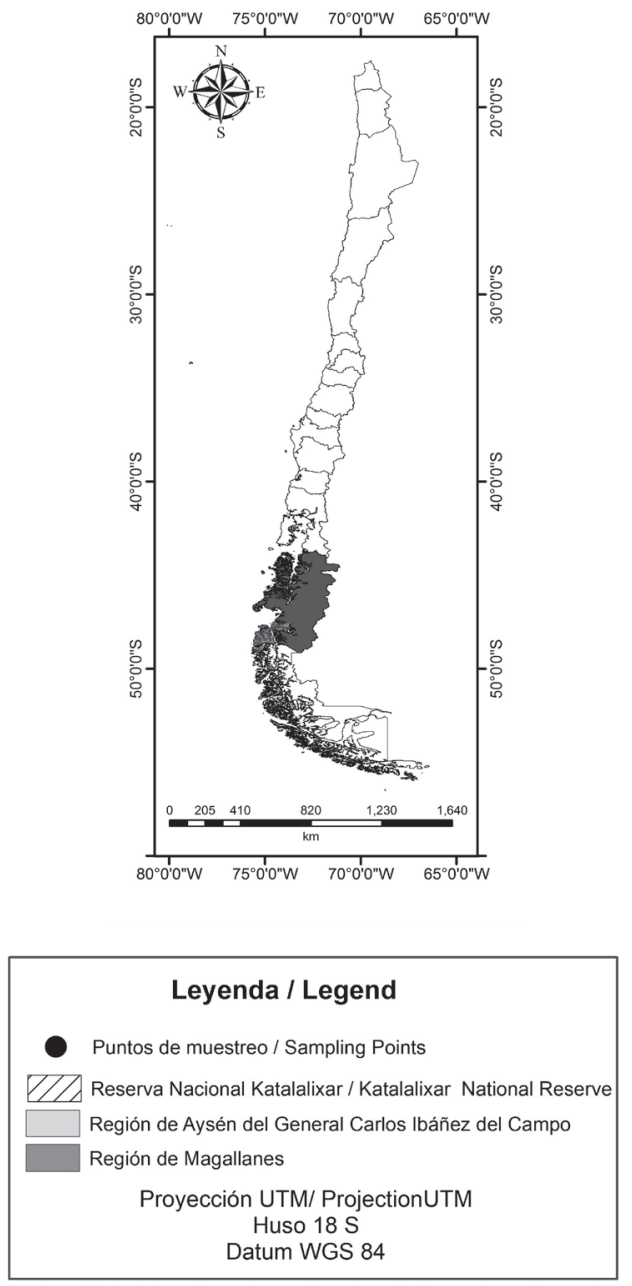

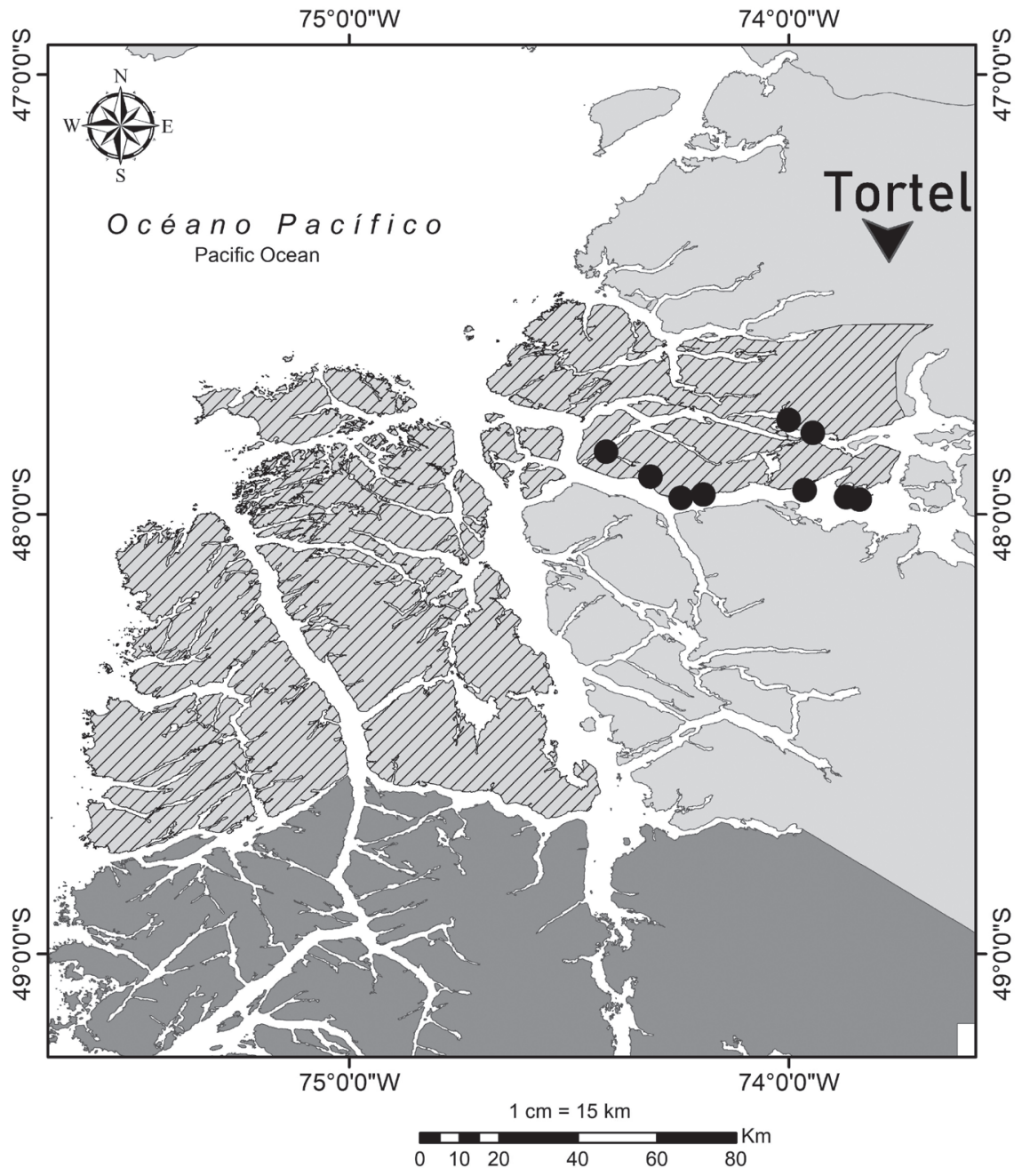

Fıgura 1. Ubicación geográfica del área de estudio en la Reserva Nacional Katalalixar, Comuna de Tortel, Región de Aysén, Chile. / Geographic location of the study area in the Katalalixar National Reserve, Tortel district, Aisen Region, Chile. 
Las familias con mayor riqueza de líquenes en la Reserva Nacional Katalalixar son Parmeliaceae con 19 especies, Lobariaceae con 17 y Pannariaceae con 8 (Fig. 3B). Los

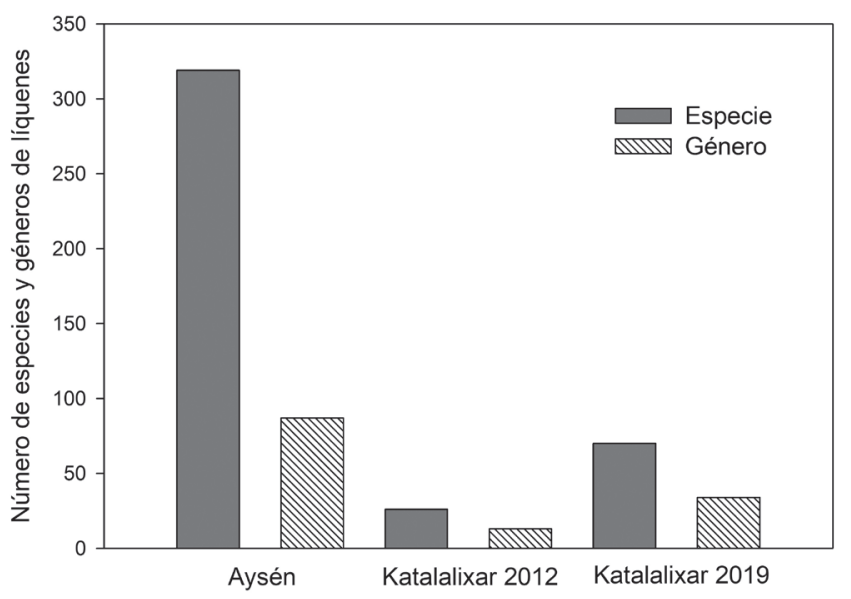

Figura 2. Número de especies y géneros de líquenes presentes en la región de Aysén y Reserva Nacional Katalalixar reportados por Quilhot et al. (2012) y el presente estudio. / Species and genera number of lichens present in Aisen Region and Katalalixar National Reserve reported by Quilhot et al. (2012) and present sutdy. géneros con mayor riqueza de líquenes son, Pseudocyphellaria con 12 especies, Menegazzia con 6 y Sticta con 5 (Fig. 3A). Las especies con mayor número de presencias en los puntos de muestreo fueron Coccotrema coccophorum, Leifidium tenerum, Pseudocyphellaria berberina, Platismatia glauca, Menegazzia opuntioides, Nephroma antarcticum y Bunodophoron australe (Tabla 1).

Respecto al tipo de fotobionte, los líquenes con algas verdes como fotobionte principal alcanzan 46 especies (66\%) frente a 24 especies (34\%) con cianobacterias o algas verdeazules (Tabla 1). Cabe destacar que en los puntos de muestreo asociados al bosque de Pilgerodendron uviferum (punto de muestreo 7) y turberas (puntos de muestreo 8 y 9) se encontró gran diversidad de especies con cianobacteria (un total de 19 especies) destacando Degelia gayana, Erioderma leylandii, Pseudocyphellaria coerulescens, P. hirsuta, P. encoensis y Sticta fuliginosa (Tabla 1). Como organismos simbióticos complejos, los líquenes son muy sensibles a los cambios ambientales siendo los cianolíquenes los más amenazados (Rubio-Salcedo et al. 2017), por lo que el registro de la riqueza de especies ayudaría a establecer comparaciones que permitan advertir cambios en la dinámica de estas comunidades liquénicas en monitoreos a largo plazo (Gatica et al. 2011).

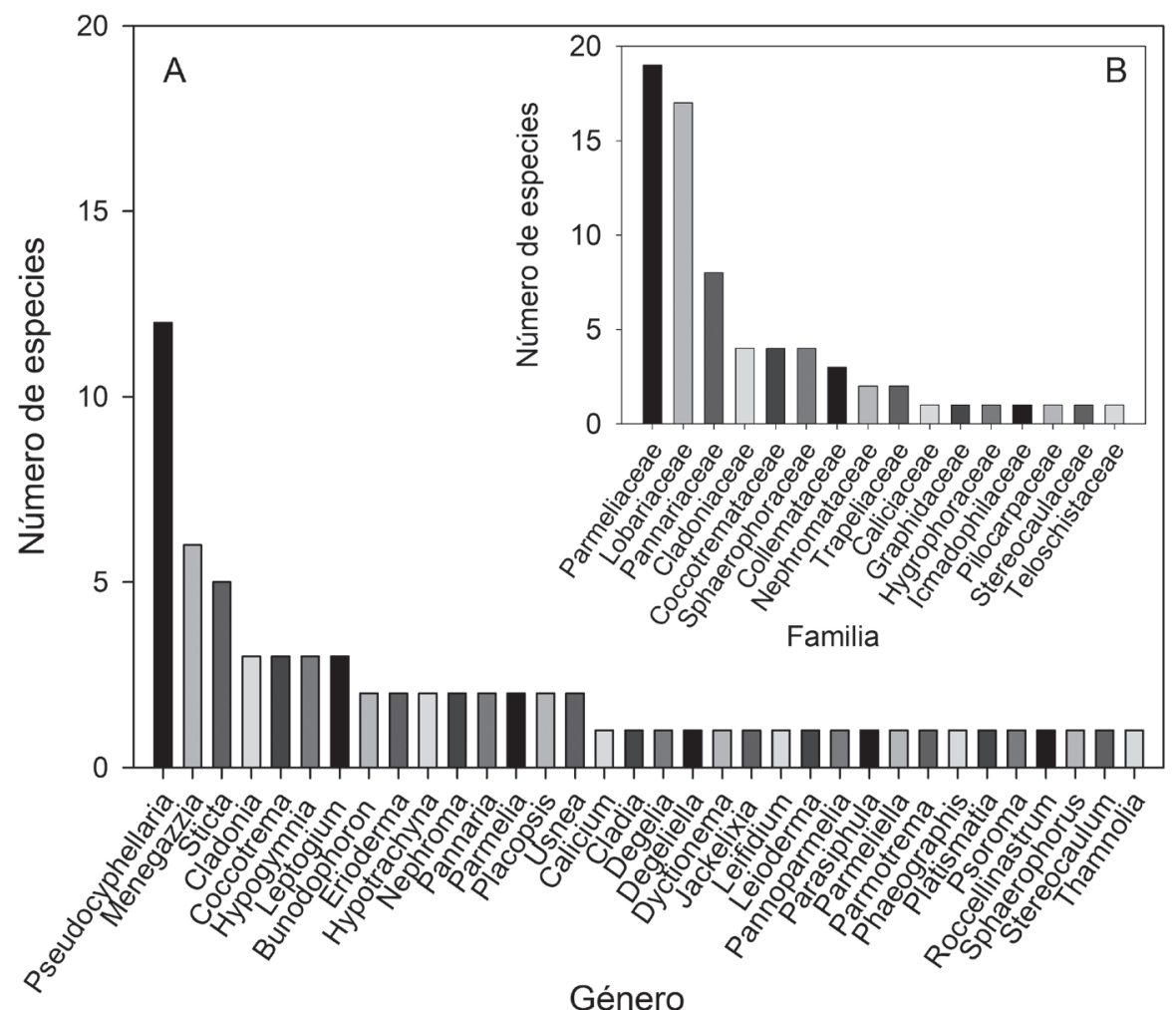

Fıgura 3. Líquenes presentes en la Reserva Nacional Katalalixar, región de Aysén. Número de especies por género (A) y familia (B). / Lichens present in Katalalixar National Reserve, Aisen Region. Number of species by genera (A) and family (B). 


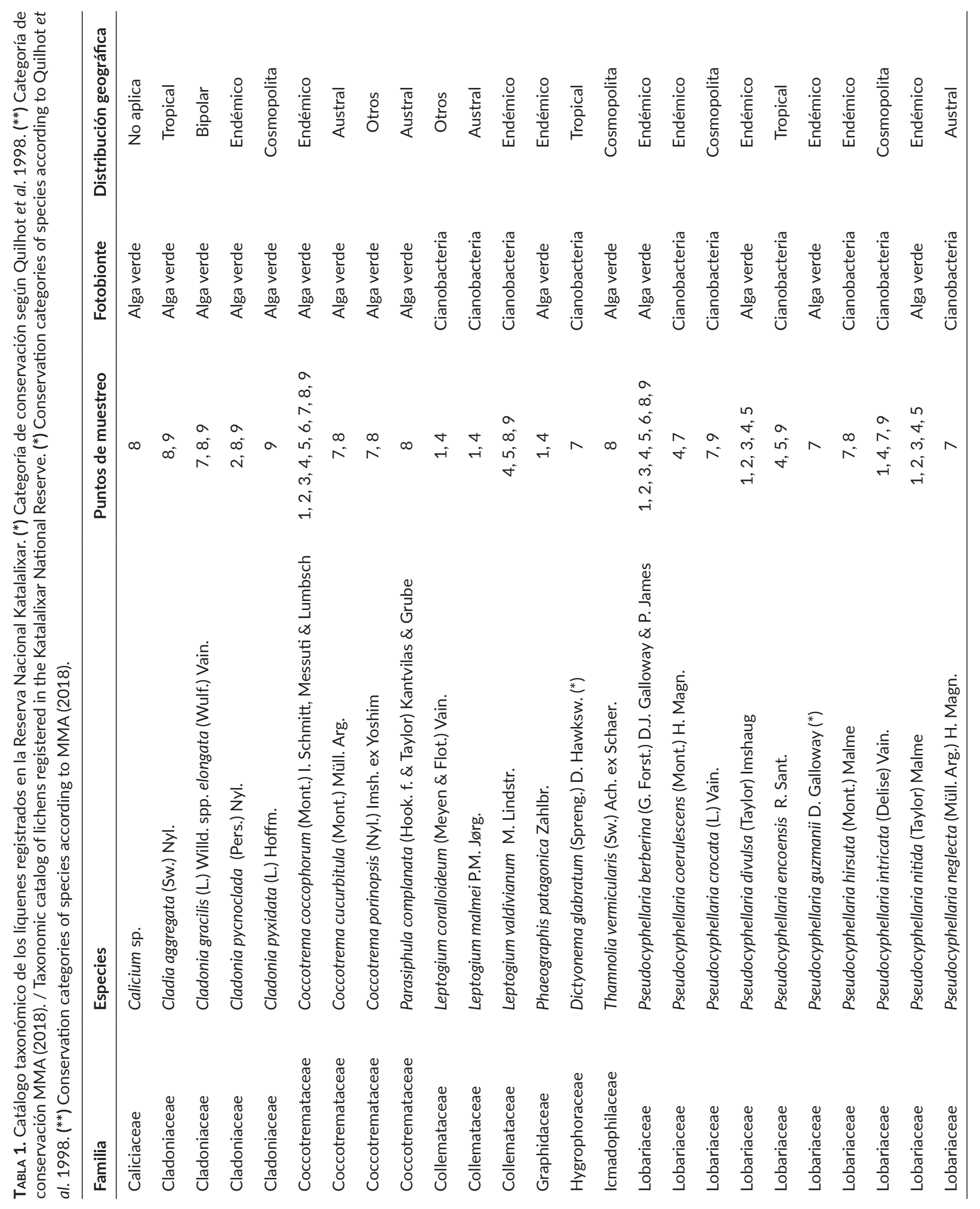




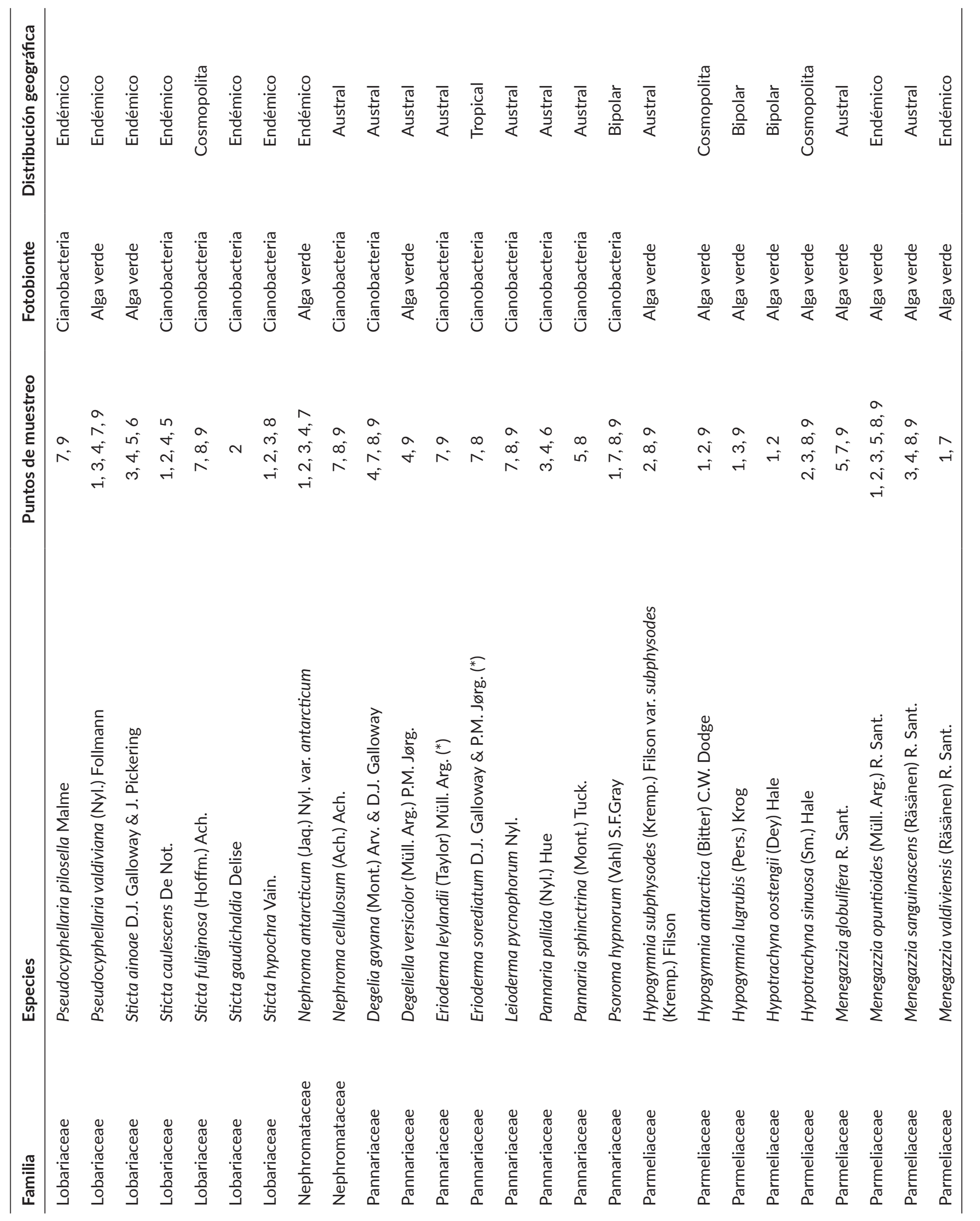




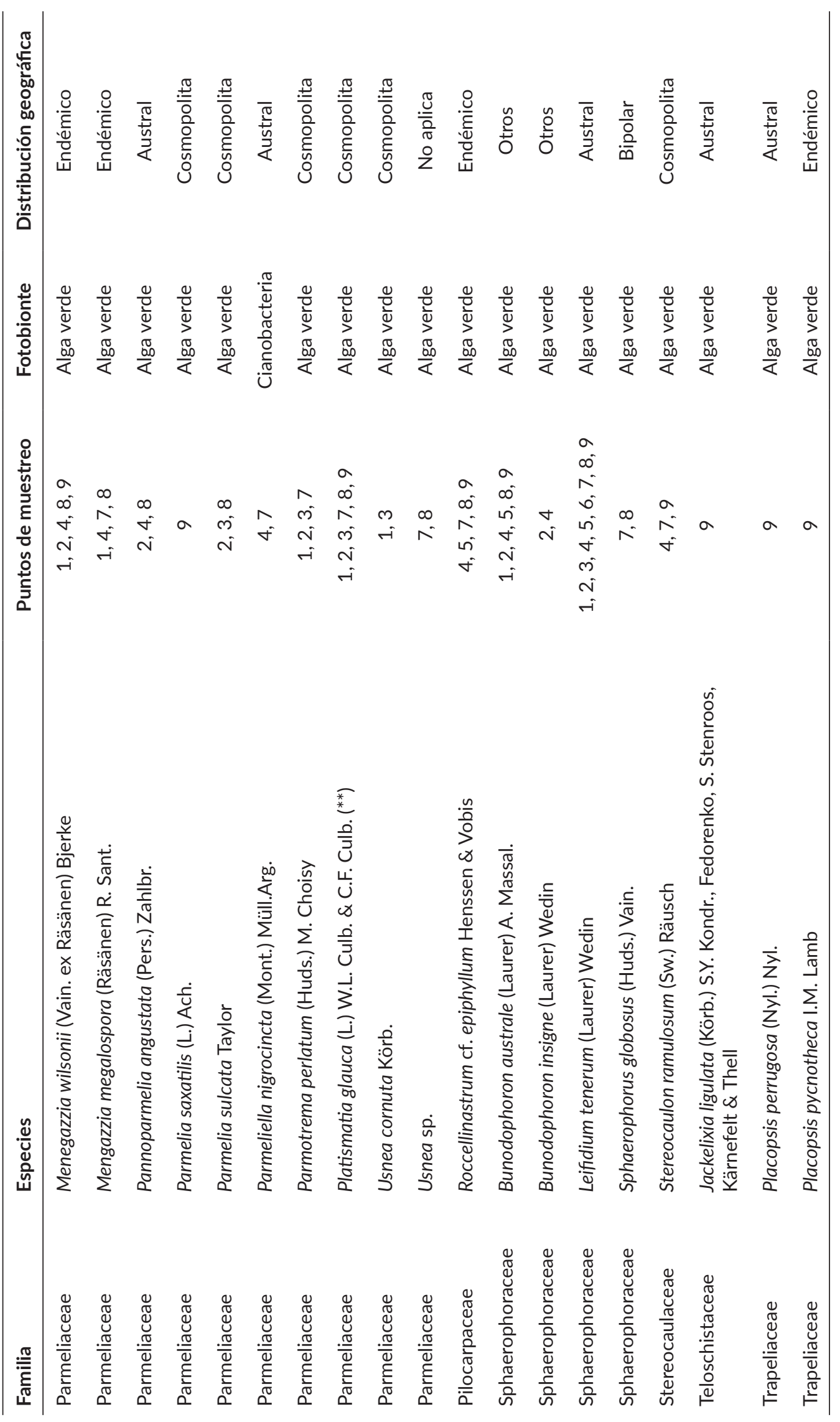


Respecto a los hábitats ocupados por los líquenes se han identificado tres grupos basados en Galloway (1992b). Una primera comunidad de especies que viven en bosques de coihue de Chiloé, con un sotobosque denso y sombrío, caracterizado por las especies Coccotrema coccophorum, Leifidium tenerum, Pseudocyphellaria berberina, P. nitida, Menegazzia opuntioides, Sticta caulescens y Nephroma antarcticum. Una segunda comunidad es la que reúne a las especies del bosque de $P$. uviferum y las especies asociadas a turberas esfagnosas, con matorral mixto. Estas se describen como una sola unidad ya que las especies diferenciales son las mismas, entre ellas Degelia gayana, Dictyonema glabratum, Platismatia glauca, Erioderma leylandii, Leioderma pycnophorum, Mengazzia megalospora, M. opuntoide, Nephroma cellulosum, Hypotrachyna sinuosa, Sticta fuliginosa, Pseudocyphellaria berberina, P. crocata y P. pilosella. La riqueza específica de líquenes asociadas a esta comunidad es alta (53 especies), coincidiendo con Villagra et al. (2009), quienes registraron 35 especies de líquenes en turberas esfagnosas asociados a forófitos leñosos, como por ejemplo: P. uvifera, Nothofagus antarctica (G.Forst.) Oerst. y Nothofagus betuloides (Mirb.) Oerst. en la comuna de Tortel. Cabe destacar el nuevo registro para la región de Aysén, de la especie endémica de Chile Pseudocyphellaria guzmanii creciendo sobre P. uvifera. Esta especie anteriormente fue registrada en la IX y $\mathrm{X}$ regiones (Galloway 1992b). En el piso de esta comunidad también se registran las especies Thamnolia vermicularis, Parasiphula complanata, Cladia aggregata y Cladonia gracilis var. elongata, esta última fue descrita por León et al. (2013) en turberas de Chiloé y también ha sido registrada previamente en turberas de Caleta Tortel (Villagra et al. 2009). Finalmente, la tercera comunidad, se caracteriza por especies que crecen notoriamente en la zona de playa, en las piedras y arena, estas especies parecen actuar como estabilizadores del suelo (Galloway 1992b). En este hábitat destacan especies como Cladonia pyxidata y Stereocaulon ramulosum, Jackelixia ligulata, Placopsis perrugosa y Parmelia saxatilis.

Desde una perspectiva biogeográfica (Tabla 1), los líquenes de la RN Katalalixar son de considerable interés. Los líquenes endémicos del sur de Sudamérica representan el 34\% (Fig. 4), destacan numerosas especies del género Pseudocyphellaria ( $P$. berberina, P. coerulescens, P. divulsa, P. guzmanii, P. hirsuta, P. nitida, P. pilosella, P. valdiviana), Sticta (S. ainoae, S. caulescens, S. gaudichaldia, S. hypochra) y Menegazzia (M. megalospora, M. opuntioides, M. valdiviensis, M. wilsonii), junto con las especies Coccotrema coccophorum, Leptogium valdivianum, Nephroma antarcticum, Phaeographis patagonica, Placopsis pycnotheca son endémicas del sur de Sudamérica. El $28 \%$ de las especies tiene distribución austral, destacan aquí especies como Leioderma pycnophorum, Coccotrema cucurbitula, Nephroma cellulosum y Parasiphula complanata. Las especies con distribución bipolar alcanzaron el 7\%, destacando las especies Cladonia gracilis, Sphaerophorus globosus, Hypogymnia subphysodes y Hypotrachyna oostingii. Las especies cosmopolitas representan el $19 \%$ y destacan aquí especies como Platismatia glauca, Sticta fuliginosa y Pseudocyphellaria crocata. En cuanto a las categorías de conservación de las especies de líquenes registradas, existen 5 casos para los que se ha reportado alguna categoría de conservación (Quilhot et al. 1998; MMA 2018). En primer lugar Dictyonema glabratum y Erioderma leylandii, consideradas Fuera de Peligro en la XI Región por Quilhot et al. (1998). Erioderma sorediatum considerada Vulnerable por Quilhot et al. (1998). Pseudocyphellaria guzmanii, especie endémica de Chile y nuevo registro para la Región de Aysén. Anteriormente ha sido descrita entre la IX y X regiones y es clasificada como Vulnerable por Quilhot et al. (1998) en toda su área de distribución debido a su escasa frecuencia y a la explotación del bosque nativo. Cabe destacar, que estas categorías (Quilhot et al. 1998) son propuestas basadas en categorías enunciadas por la IUCN pero no forman parte de un cuerpo legal dentro del listado de especies con alguna categoría de conservación del Ministerio del Medio Ambiente. En este sentido solo Platismatia glauca presenta una categoría de conservación, según Reglamento de Clasificación de Especies Silvestres del Ministerio del Medio Ambiente (MMA 2018) como Preocupación Menor (LC). La principal amenaza, para estas cinco especies es la disminución de la cobertura del bosque templado lluvioso por cambio de uso de suelo o explotación forestal.

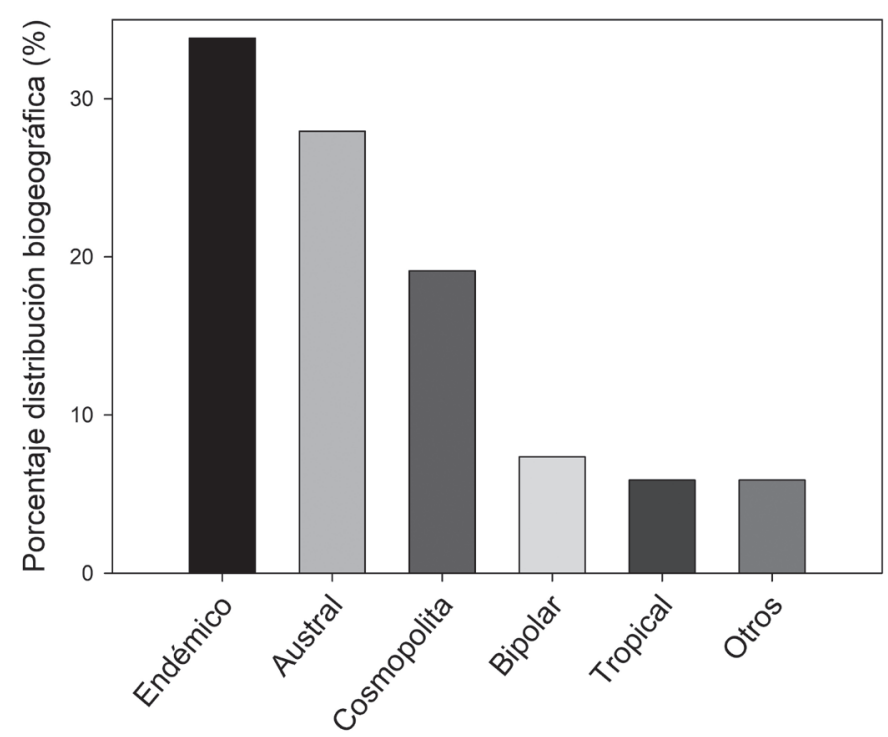

Figura 4. Porcentaje de la distribución biogeográfica de las especies de la Reserva Nacional Katalalixar. / Percentage of the biogeographical distribution of the species of the Katalalixar National Reserve. 


\section{CONCLUSIONES}

La biota liquénica registrada se caracteriza por una alta diversidad y alto grado de endemismo, destacando la importancia de los bosques de Nothofagus nitida, Pilgerodendron uviferum y turberas de la Patagonia chilena como herramienta para la conservación de estos organismos. Una elevada proporción de las especies reportadas en este trabajo son endemismos del sur de Sudamérica (34\%) y en conjunto con las especies de distribución austral representan más de la mitad de las especies halladas en este trabajo.

\section{AGRADECIMIENTOS}

A ONG OCEANA por financiar la expedición y el acceso a la información. A Fundación Fungi, Raúl Pereda (guardaparque CONAF) y Sebastián Vega quien elaboró el mapa. Jacob Blaustein Center for Scientific Cooperation del Jacob Blaustein Institutes for Desert Research por la beca postdoctoral de DA.

\section{REFERENCIAS}

Allen, J.L., Lendemer, J.C. 2016. Climate change impacts on endemic, high-elevation lichens in a biodiversity hotspot. Biodiversity and Conservation 25(3): 555-568.

Amigo, J. Ramírez, C. 1998. A bioclimatic classification of Chile: woodland communities in the temperate zone. Plant Ecology 136(1): 9-26.

Arnold, A.E., Miadlikowska, J., Higgins, K.L., Sarvate, S.D., Gugger, P., Way, A., Hofstetter, V., Kauff, F., Lutzoni, F. 2009. A phylogenetic estimation of trophic transition networks for ascomycetous fungi: are lichens cradles of symbiotrophic fungal diversification?. Systematic Biology 58(3): 283-297.

Asplund, J., Wardle, D.A. 2016. How lichens impact on terrestrial community and ecosystem properties. Biological Reviews 92(3): 1720-1738.

Bjerke, J., Elvebakk, A., Quilhot, W. 2003. Distribución y ecología de las especies sorediosas de Menegazzia (Parmeliaceae, Ascomycota liquenizado) en Chile. Revista Chilena de Historia Natural 76(1): 79-98.

Bjerke, J. 2005. Synopsis of the lichen genus Menegazzia (Parmeliaceae, Ascomycota) in South America. Mycotaxon 91: 423-454.

Brodo, I., Sharnoff, M., Sharnoff, S. 2001. Lichens of North America. Yale University Press. 795 pp.

Casanova-Katny, A., Barták, M., Gutierrez, C. 2019. Open top chamber microclimate may limit photosynthetic processes in Antarctic lichen: Case study from King George Island,
Antarctica. Czech Polar Reports 9(1): 61-77.

Ellis, C.J. 2013. A risk-based model of climate change threat: hazard, exposure and vulnerability in the ecology of lichen epiphytes. Botany 91(1): 1-11.

Galloway, D. 1985. Flora of New Zealand Lichens. P.S. Hasselberg, New Zealand Government Printer, Wellington. 662 pp.

Galloway, D.J. 1991. Phytogeography of Southern Hemisphere Lichens. In: Nimis, P.L., Crovello, T.J. (Eds.) Quantitative approaches to phytogeography: 233-262. Tasks for vegetation science, vol 24. Springer, Dordrecht.

Galloway, D.J. 1992a. Studies in Pseudocyphellaria (lichens) III. The South American species. Bibliotheca Lichenologica. Berlin-Stuttgart. 275 pp.

Galloway, D.J. 1992b. Lichens of Laguna San Rafael, Parque Nacional, Southern Chile, indicators of environmental change. Global Geology and Biogeography Letters 2(2): 37-45.

Galloway, D.J. 1994. Studies on the lichen genus Sticta (Schrreber) Ach. I. Southern South American species. Lichenologist 26: 223-282.

Galloway, D.J. 1996. Los líquenes del bosque templado de Chile. En: Armesto, J.J., Villagrán, C., Arroyo, M.T.K. (Eds.) Ecología de los bosques nativos de Chile: 101-112. Editorial Universitaria, Universidad de Chile, Chile.

Galloway, D.J. 2008. Lichen biogeography. In: Nash, T.H. (Ed.). Lichen Biology: 315-335. Second Edition. Cambridge, Cambridge University Press.

Gatica, A, Pereira, I, Vallejos, O. 2011. Líquenes epífitos: una herramienta para estudiar la continuidad ecológica en Isla Mocha, Chile. Gayana Botánica 68(2): 226-235.

Giordani, P., Brunialti, G. 2015. Sampling and Interpreting Lichen Diversity Data for Biomonitoring Purposes. In: Upreti, D., Divakar, P., Shukla, V., Bajpai, R. (Eds.) Recent Advances in Lichenology: 19-46. Springer, New Delhi.

Hajek, E., Di Castri, F. 1975. Bioclimatografía de Chile. Ediciones Universidad Católica de Chile, Santiago. 114 pp.

Hedenäs, H., Ericson, L. 2000. Epiphytic macrolichens as conservation indicators: successional sequence in Populus tremula stands. Biological conservation 93(1): 43-53.

Hodkinson, B.P, Lutzoni, F. 2009. A microbiotic survey of lichenassociated bacteria reveals a new lineage from the Rhizobiales. Symbiosis 49: 163-180.

Humphrey, J.W., Davey, S., Peace, A.J., Ferris, R., Harding, K. 2002. Lichens and bryophyte communities of planted and semi-natural forests in Britain: the influence of site type, stand structure and deadwood. Biological Conservation 107(2): 165-180.

Jentsch, A., Beierkuhnlein, C., White, P. 2002. Scale, the dynamic stability of forest ecosystems, and the persistence of biodiversity. Silva Fennica 36(1): 393-400.

Jørgensen, P.M., Galloway, D.J. 1992. Pannariaceae. Flora of Australia 54: 246-293. 
Kuusinen, M., Siitonen, J. 1998. Epiphytic lichen diversity in old-growth and managed Picea abies stands in southern Finland. Journal of Vegetation Science 9(2): 283-292.

Lendemer, J.C., Allen, J.L. 2014. Lichen biodiversity under threat from sea-level rise in the Atlantic Coastal. Bioscience 64(10): 923-931.

León, C.A., Oliván, G., Pino-Bodas, R. 2013. New distributional records for Chilean bryophyte and lichen flora. Gayana Botanica 70(2): 241-246.

Luebert, F., Pliscoff, P. 2006. Sinopsis bioclimática y vegetacional de Chile. Editorial Universitaria, Santiago. 316 pp.

Martínez, I., Aragón, G., Sarrión, F.J., Escudero, A., Burgaz, A.R., Coppins, B.J. 2003. Threatened lichens in central Spain (saxicolous species excluded). Cryptogamie Mycologie 24: 73-97.

McCune, B. 2000. Lichen communities as indicators of forest health. The Briologist 103(2): 353-356.

Nash III, T.H., Gries, C., Elix, J.A. 1995. A revision of the lichen genus Xanthoparmelia in South America. Bibliotheca Lichenologica 56: 1-157.

Nash III, T.H., Sipman, H.G.M., Elix, J.A. 2002. Hypotrachyna. In: Nash III, T.H., Ryan, B.D., Gries, C., Bungartz, F. (Eds.) Lichen Flora of the Sonoran Desert Region Vol. 1: 238251. Lichens Unlimited, Arizona State University, Tempe, Arizona.

Nash III, T.H., Elix, J.A. 2002a. Flavoparmelia. In: Nash III, T.H., Ryan, B.D., Gries, C., Bungartz, F. (Eds.) Lichen Flora of the Sonoran Desert Region Vol. 1: 194-196. Lichens Unlimited, Arizona State University, Tempe, Arizona.

Nash III, T.H., Elix, J.A. 2002b. Parmotrema. In: Nash III, T.H., Ryan, B.D., Gries, C., Bungartz, F. (Eds.) Lichen Flora of the Sonoran Desert Region Vol. 1: 316-339. Lichens Unlimited, Arizona State University, Tempe, Arizona.

Nash III, T.H., Elix, J.A. 2002c. Rimelia. In: Nash III, T.H., Ryan, B.D., Gries, C., Bungartz, F. (Eds.) Lichen Flora of the Sonoran Desert Region Vol. 1: 449-451. Lichens Unlimited, Arizona State University, Tempe, Arizona.

Nylander, W. 1866. "Hypochlorite of Lime and Hydrate of Potash, two new Criteria in the study of Lichens". Journal of the Linnean Society of London, Botany 9 (38): 358-365.

Paillet, Y, Bergès, L., Hjältén, J., Odor, P., Avon, C., BernhardtRömermann, M, Bijlsma, R.J., De Bruyn, L., Fuhr, M., Grandin, U., Kanka, R., Lundin, L., Luque, S., Magura, T., Matesanz, S., Mészáros, I., Sebastià, M.T., Schmidt, W., Standovár, T., Tóthmérész, B., Uotila, A., Valladares, F., Vellak, K., Virtanen, R. 2010. Biodiversity differences between managed and unmanaged forests: meta-analysis of species richness in Europe. Conservation Biology 24(1): 101-112.

Pharo, E.J., Beattie, A.J., Binns, D. 1999. Vascular Plant Diversity as a Surrogate for Bryophyte and Lichen Diversity. Conservation Biology 13(2): 282-292.

Quilhot, W., Pereira, I., Guzmán, G., Rodríguez, R., Serey, I. 1998. Categorías de conservación de líquenes nativos de Chile. Boletín del Museo Nacional de Historia Natural 47: 9-22.

Quilhot, W., Cuellar, M., Díaz, R., Riquelme, F., Rubio, C. 2012. Lichens of Aisen, Southern Chile. Gayana Botanica 69(1): 57-87.

Richardson, D., Cameron, R. 2009. Cyanolichens: Their response to pollution and possible management strategies for their conservation in northeastern North America. Northeastern Naturalist 11(1): 1-22.

Rivas-Plata, E., Lücking, R., Lumbsch, H.T. 2008. When family matters: an analysis of Thelotremataceae (Lichenized Ascomycota: Ostropales) as bioindicators of ecological continuity in tropical forests. Biodiversity and Conservation 17(6): 1319-1351.

Rubio, C., Saavedra, M., Cuéllar, M., Díaz, R., Quilhot, W. 2013. Epiphytic lichens of Conguillío National Park, Southern Chile. Gayana Botanica 70(1): 66-81.

Rubio-Salcedo, M., Psomas, A., Prieto, M., Zimmermann, E., Martinez, I. 2017. Case study of the implications of climate change for lichen diversity and distributions. Biodiversity and Conservation 26(5): 1121-1141.

Scherhag, R. 1969. Klimatologie. Georg Westermann Verlag. Braunschweig. 165 pp.

Seremi MMA-Aysén. 2018. Estrategia regional de Biodiversidad 2015-2030, región de Aysén del General Carlos Ibáñez del Campo. Documento técnico de la Secretaría Regional Ministerial de Medio Ambiente, región de Aysén, financiado por el Gobierno Regional y su Consejo, bajo el Plan Especial de Zonas Extremas (PEDZE). 80 pp.

Sillett, S.C. 1994. Growth rates of two epiphytic cyanolichen species at the edge and in the interior of a 700-year-old Douglas-firforest in the western Cascades of Oregon. Bryologist 97(3): 321-324.

Stenross, S. 1995. Cladoniaceae (Lecanorales, Ascomycotina Liquenizada), en la flora de Chile. Gayana Botanica 52: 89-131.

Villagra, J., Montenegro, D., San Martín, C., Ramírez, C., Álvarez, I. 2009. Estudio de la flora liquénica de las turberas de la comuna de Tortel (región de Aysén), Patagonia chilena. Anales del Instituto de la Patagonia 37(1): 53-62.

Villagrán, C., Hinojosa, F. 1997. Historia de los bosques de Sudamérica II. Fitogeografía. Revista Chilena de Historia Natural 70: 241-267.

Wedin, M. 1995. The lichen family Sphaerophoraceae (Caliciales, Ascomycotina) in temperate areas of the Southern hemisphere. Acta Universitattis Upsaliensis, Symbolae Botanicae Upsalienses 31: 1.

Received: 26.07.2019

Accepted: 19.03.2020 